\title{
Imaging Fluorescently Stained DNA with CCD Technology How to Increase Sensitivity and Reduce Integration Times
}

\author{
Dr. Paru Oatey \\ Syngene, Beacon House, Nuffield Road, Cambridge, CB4 1TF, UK. Tel: +44(0) 1223-727123 Fax: +44 (0) 1223-727101 \\ Email: paru.oatey@syngene.com \\ Web site: www.syngene.com
}

\section{INTRODUCTION}

Ethidium bromide has long been the method of choice for staining DNA and RNA in agarose gels. However, this dye has the disadvantages of being mutagenic and toxic. To improve user safety, companies have developed alternative fluorescent dyes such as the SYBR ${ }^{\circledR}$ range of dyes ${ }^{1,2}$ which offer a better safety profile ${ }^{3}$. The complaint of many researchers is that in the case of the SYBR ${ }^{\circledR}$ Safe stain it does not offer the same level of sensitivity as ethidium bromide and with $\mathrm{SYBR}^{\circledR}$ Green stain and $\mathrm{SYBR}^{\circledR}$ Gold stain can require longer integration times for visualisation, when viewed with UV light.

UV transilluminators commonly used to excite SYBR dyes, emit UV in a broad band (approximately 100nm wide) centred at 300nm. As most fluorescent dyes have a bimodal excitation spectrum, with one excitation peak in the UV and another in the visible range, using a blue converter screen to change UV to light with an excitation range of 410-510nm, where SYBR dyes have a second excitation peak, is said to improve the sensitivity of many SYBR dyes.

In this article we describe how using CCD technology with a blue converter screen and the correct lighting and filter conditions enables scientists to rapidly image SYBR Green and SYBR Gold stains with better levels of sensitivity than DNA stained with ethidium bromide, thus offering a safe yet accurate imaging method.

\section{MATERIALS AND METHODS}

Into an agarose gel $(0.8 \%)$ containing ethidium bromide $(0.5 \mu \mathrm{g} / \mathrm{ml})$ and three agarose gels containing no dye, the following amounts of HyperLadder DNA marker (Bioline, London, UK) were loaded: $50,25,10,5,1,0.5,0.25$, $0.1,0.05,0.01 \mathrm{ng}$. This marker contains 14 bands ranging in size from 200bp - 10000bp and the titration amount was based on the 1000bp band. The gels were run under standard electrophoresis conditions and the three gels that did not contain ethidium bromide were stained with either, SYBR Safe, SYBR Green or SYBR Gold stains (Invitrogen, Paisley, UK) according to manufacturers' protocols.

The gels were then transferred to a G:BOX Chemi HR16 (Syngene, Cambridge, UK) for imaging. This system was chosen because it has a 16-bit, high resolution camera which can be fitted with a range of emission filters. The camera is integrated to a computer controlled light-tight darkroom that has overhead Epi-UV and Epi-Blue light and can house a dual wavelength UV transilluminator, making the system suitable for imaging any commercial fluorescent dye.

To produce an optimum image of the gels stained with SYBR dyes and ethidium bromide, images of the gels were captured in the CCD system using a range of lighting, screen and filter combinations. These consisted of medium wave UV (275-375nm with a peak at $302 \mathrm{~nm})$, Epi-Blue (488nm), a blue light converter screen and one of the short pass (SP), UV (mid pass) or SG blue light emission filters.

The results of these imaging studies are listed in Table 1.

\section{RESULTS AND DISCUSSION}

With the CCD system, using medium wave UV and a mid pass filter (the optimum, established imaging conditions for ethidium bromide) allows visualization of $0.5 \mathrm{ng}$ of the 1000bp DNA fragment stained with ethidium bromide (Figure 1) in $35 \mathrm{~ms}$.

For the gel stained with SYBR Gold dye, medium wave UV, a blue light converter screen and an SG filter produces optimum results, allowing visualization of $0.1 \mathrm{ng}$ of the 1000bp DNA fragment (Figure 2) in 3.5s. The best results for imaging gels stained with SYBR Green dye were obtained using medium wave UV, a blue light converter screen and an SG filter, permitting visualization of $0.1 \mathrm{ng}$ of the 1000bp fragment (Figure 3) in 0.5s.

Using gels stained with SYBR Safe dye, medium wave UV and an SP filter provides the best results, with visualization of $0.5 \mathrm{ng}$ of the $1000 \mathrm{bp}$ DNA fragment (Figure 4) in 130ms.

The results show that given the lighting conditions, screen and correct emission filter, CCD technology can achieve levels of sensitivity, which are within the reported range of sensitivity limits of 60-100pg for both SYBR Green and SYBR Gold stains and 500ng for SYBR Safe ${ }^{4}$ stain yet without long integration times.

By using a blue light converter screen and an SG filter the sensitivity of the CCD system with SYBR Gold and SYBR Green stains can be doubled making these dyes five times more sensitive than ethidium bromide. An additional advantage of using these imaging conditions with SYBR 


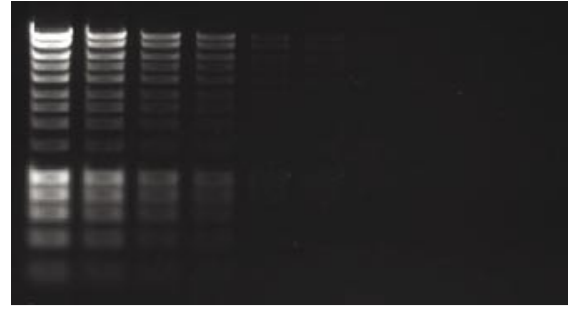

Figure 1. Agarose gel stained with ethidium bromide showing HyperLadder DNA marker from right to left, the top 1000 bp band is seen in the following amounts: 50, 25, 10, 5, 1, 0.5, 0.25, $0.1,0.05,0.01 \mathrm{ng}$. The gel image was captured with CCD-technology using a medium wave transilluminator and mid pass UV filter.

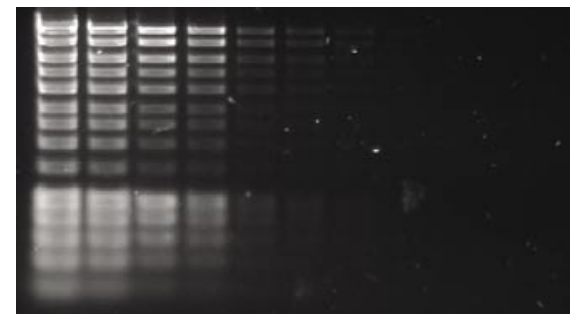

Figure 3. Agarose gel stained with SYBR Green dye showing HyperLadder DNA marker from right to left, the top $1000 \mathrm{bp}$ band is seen in the following amounts: $50,25,10,5,1,0.5,0.25,0.1,0.05$, $0.01 \mathrm{ng}$. The gel image was captured with CCD-technology using a medium wave transilluminator, a blue light converter and SG filter.

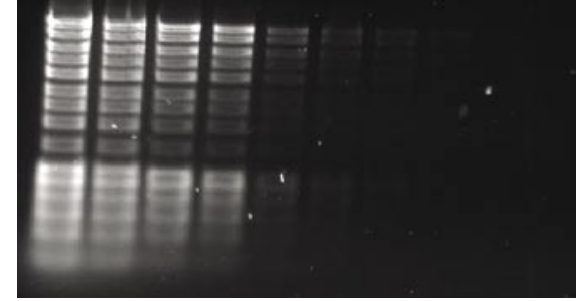

Figure 2. Agarose gel stained with SYBR Gold dye showing HyperLadder DNA marker from right to left, the top $1000 \mathrm{bp}$ band is seen in the following amounts: $50,25,10,5,1,0.5,0.25,0.1,0.05$, $0.01 \mathrm{ng}$. The gel image was captured with CCD-technology using a medium wave transilluminator, a blue light converter and SG filter.

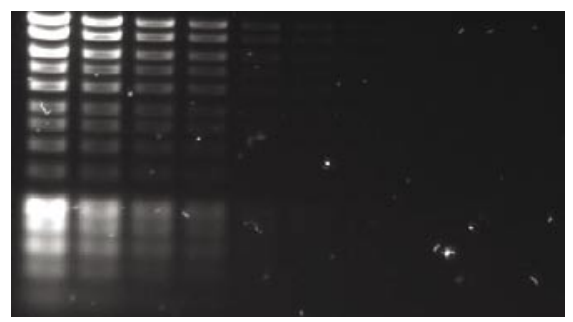

Figure 4. Agarose gel stained with SYBR Safe dye showing HyperLadder DNA marker from right to left, the top $1000 \mathrm{bp}$ band is seen in the following amounts: $50,25,10,5,1,0.5,0.25,0.1,0.05$, $0.01 \mathrm{ng}$. The gel image was captured with CCD-technology using a medium wave transilluminator and SP filter.

Table 1. Conditions used to image fluorescent dyes using a G:BOX Chemi HR16 CCD based system.

\begin{tabular}{|l|c|c|c|c|c|}
\hline Nucleic Acid Stain & Excitation Source & Screen & Emission Filter & Integration Time & Amount of 1000bp band detected \\
\hline Ethidium Bromide & UV & None & UV & $35 \mathrm{~ms}$ & $0.5 \mathrm{ng}$ \\
\hline SYBR Gold & UV & Blue light converter & SG filter & $3.5 \mathrm{~s}$ & $0.1 \mathrm{ng}$ \\
\hline & Epi-Blue & None & SG filter & $9 \mathrm{~s}$ & $0.25 \mathrm{ng}$ \\
\hline & UV & None & SP filter & $55 \mathrm{~ms}$ & $0.25 \mathrm{ng}$ \\
\hline & UV & Blue light converter & SG filter & $500 \mathrm{~ms}$ & $0.1 \mathrm{ng}$ \\
\hline & Epi-Blue & None & SG filter & $15 \mathrm{~s}$ & $0.1 \mathrm{ng}$ \\
\hline SYBR Safe & UV & None & SP filter & $90 \mathrm{~ms}$ & $0.25 \mathrm{ng}$ \\
\hline & UV & Blue light converter & SG filter & $7.5 \mathrm{~s}$ & $0.5 \mathrm{ng}$ \\
\hline & Epi-Blue & None & SG filter & $20 \mathrm{~s}$ & $0.5 \mathrm{ng}$ \\
\hline
\end{tabular}

Green stain is the integration time is halved, to make it comparable with that used to image ethidium bromide and means SYBR Green stain is a good best choice for rapid and accurate DNA quantification.

For SYBR Safe stain, using medium wave UV light and a SP filter ensures that sensitivity is the same as ethidium bromide with a comparable integration time. Therefore, if sensitivity is not a major requirement but using a non-mutagenic and non toxic stain is, then these imaging conditions for SYBR Safe stain will deliver the required results.

\section{CONCLUSIONS}

Imaging fluorescent dyes with high sensitivity and short integration times is possible with CCD-based systems, which have a high performance camera capable of detecting a wide range of emission wavelengths. Additionally, the system has to be able to utilise different lighting, screens and emission filters to achieve the right combination of il- lumination and detection. Therefore, because the G:BOX Chemi HR16 provides these flexible capabilities, it offers an excellent method for rapid yet safe imaging of fluorescently stained DNA.

\section{ACKNOWLEDGEMENTS}

We thank Matt Beaudet of Invitrogen for the generous donation of SYBR dyes used in this work and for his input and advice in the preparation of this article. We also thank Xiaolan Yu and Paul Dupree, Department of Biochemistry, University of Cambridge for preparing the agarose gels used in this work.

\section{REFERENCES}

1. Biotechniques. 27(1):34-6, (1999).

2. Anal. Biochem. 268(2):278-88, (1999).

3. Mutat. Res. 2; 439(1):37-47, (1999).

4. www.invitrogen.com 